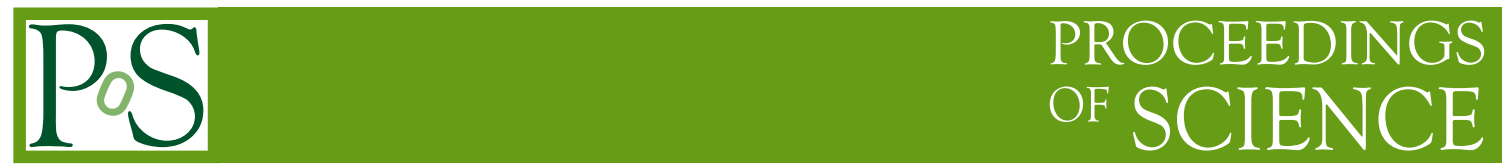

\title{
Analysis of isospin symmetry for fragmentation functions
}

\author{
Kai-bao Chen $^{a, *}$ \\ ${ }^{a}$ School of Science, Shandong Jianzhu University, \\ Jinan, Shandong 250101, China
}

E-mail: chenkaibao19@sdjzu.edu.cn

We make an analysis of the isospin symmetry for fragmentation functions assuming isospin symmetry in strong interactions. Taking hadron decay contributions into account, we show that the isospin symmetry is held for unpolarized $\Lambda$ fragmentation functions. Only tiny violations are allowed for the polarized $\Lambda$ fragmentation functions. We show that the recent Belle data on the transverse polarization of $\Lambda$ can be reproduced if the isospin symmetry is kept in the parameterization of the corresponding polarized fragmentation functions. This isospin symmetry can be checked in future SIDIS experiments with different nucleus targets.

*** Particles and Nuclei International Conference - PANIC2021 ***

*** 5 - 10 September, $2021 * * *$

$* * *$ Online $* * *$

${ }^{*}$ Speaker 


\section{Introduction}

Fragmentation functions (FFs) are important quantities in describing high energy reactions such as electron positron annihilation or semi-inclusive deep inelastic scattering. The fragmentation processes are dominated by strong interaction. Isospin symmetry is one of the fundamental properties of strong interaction, so one may naturally think that FFs will respect isospin symmetry. However, there are hadron electroweak decay contributions in the fragmentation processes which will violate isospin symmetry in general. Therefore, isospin symmetry for FFs can be violated.

Recently, the Belle collaboration has carried out a nice measurement on the transverse polarization of $\Lambda$ hyperon [1]. The data show a very different trend of $\Lambda$ polarization in the processes $e^{+} e^{-} \rightarrow \Lambda h^{+}$and $e^{+} e^{-} \rightarrow \Lambda h^{-}(h=\pi, K)$. The data might be a hint that the polarized FFs of $\Lambda$ have a large isospin asymmetry, because these two processes are related to each other with the exchange of $u$ and $d$ quarks in the final state. Parameterizations have been carried out by different groups [2, 3] using polarized transverse momentum dependent (TMD) FFs $D_{1 T q}^{\perp \Lambda}\left(z, p_{T}\right)$. Both the parameterizations get opposite signs between $D_{1 T u}^{\perp \Lambda}$ and $D_{1 T d}^{\perp \Lambda}$, which shows a large isospin symmetry violation for the corresponding TMD FFs. Therefore, it is very important to study: i) To what extent the isospin symmetry violation can be accommodated in FFs if it's held in QCD; ii) Do the Belle data really mean that there is a large isospin symmetry violation of $\Lambda$ FFs?

In this work, we make a systematic study of the isospin symmetry of FFs by taking resonance decays into account [4]. We also demonstrate that the Belle data on the transverse polarization of $\Lambda$ can be well described within isospin symmetric FFs.

\section{Isospin symmetry of fragmentation functions}

Unpolarized FFs. The fragmentation process $q \rightarrow h+X$ in the unpolarized case can be divided into two parts: the directly produced and the decay contribution parts, i.e.,

$$
\begin{aligned}
& D_{1 q}^{h}(z)=D_{1 q}^{h, \operatorname{dir}}(z)+D_{1 q}^{h, \text { dec }}(z), \\
& D_{1 q}^{h, \operatorname{dec}}(z)=\sum_{h_{j}} D_{1 q}^{h, h_{j}}(z) \\
& D_{1 q}^{h, h_{j}}(z)=B r\left(h, h_{j}\right) \int d z^{\prime} K_{h, h_{j}}\left(z, z^{\prime}\right) D_{1 q}^{h_{j}}\left(z^{\prime}\right),
\end{aligned}
$$

$D_{1 q}^{h, h_{j}}(z)$ is that from the decay channel $h_{j} \rightarrow h X$. The directly produced part is isospin symmetric. For the decay contribution part, we limit ourselves to light flavors and consider $J^{P}=0^{-}$pseudoscalar and $J^{P}=1^{-}$vector mesons, and $J^{P}=(1 / 2)^{+}$octet and $J^{P}=(3 / 2)^{+}$decuplet baryons. Other higher excited resonance states are just included into the directly produced part effectively due to their small production rates and the strong decay properties.

Taking $\Xi$ production as an example, we have decay contributions from $\Xi^{*}$ and $\Omega^{-}$, i.e.,

$$
D_{1 q}^{\Xi^{i}}(z)=D_{1 q}^{\Xi^{i}, \operatorname{dir}}(z)+D_{1 q}^{\Xi^{i}, \Xi^{*}}(z)+D_{1 q}^{\Xi^{i}, \Omega^{-}}(z) .
$$

Here, $i$ specifies one of the charge states and $D_{1 q}^{\Xi^{i}, \Xi^{*}}(z) \equiv \sum_{j} D_{1 q}^{\Xi^{i}, \Xi^{* j}}(z)$ is the sum of contributions from different charge states. The isospin symmetry violation is defined by $\delta D_{1 q}^{\Xi}(z) \equiv$ 
$D_{1 u}^{\Xi^{0}}(z)-D_{1 d}^{\Xi^{-}}(z)$. Under the isospin symmetry for strong interaction, we have $D_{1 u}^{\Xi^{0}, \text { dir }}(z)=$ $D_{1 d}^{\Xi^{-} \text {,dir }}(z), D_{1 u}^{\Xi^{0}, \Xi^{*}}(z)=D_{1 d}^{\Xi^{-}, \Xi^{*}}(z)$ and $D_{1 u}^{\Omega^{-}}(z)=D_{1 d}^{\Omega^{-}}(z)$. There is a small branch ratio difference, $\operatorname{\delta Br}\left(\Xi, \Omega^{-}\right) \approx 15 \%$ [5], for the weak decays $\Omega^{-} \rightarrow \Xi^{0} \pi^{-}$and $\Omega^{-} \rightarrow \Xi^{-} \pi^{0}$. This leads to a small isospin symmetry violation of $\Xi$ production given by

$$
\delta D_{1 q}^{\Xi}(z)=\delta D_{1 q}^{\Xi, \Omega^{-}}(z)=\delta B r\left(\Xi, \Omega^{-}\right) \int d z^{\prime} K_{\Xi, \Omega^{-}}\left(z, z^{\prime}\right) D_{1 u}^{\Omega^{-}}\left(z^{\prime}\right) .
$$

However, the violation is tiny because the total contribution from $\Omega^{-}$decay to $\Xi$ in the final state is only a very small fraction.

For $\Lambda$ production, it receives more decay contributions, i.e.,

$$
D_{1 q}^{\Lambda}(z)=D_{1 q}^{\Lambda, \operatorname{dir}}(z)+D_{1 q}^{\Lambda, \Omega^{-}}(z)+D_{1 q}^{\Lambda, \Sigma^{*}}(z)+D_{1 q}^{\Lambda, \Xi}(z)+D_{1 q}^{\Lambda, \Sigma^{0}}(z) .
$$

We note that for the contribution from the decay of $\Xi$, although there is a small isospin symmetry violation for $\Xi$ production, it does not lead to isospin symmetry violation in the $\Lambda$ production. The reason is that both $\Xi^{0}$ and $\Xi^{-}$contribute equally to $\Lambda$ production via the weak decays of $\Xi \rightarrow \Lambda \pi$. The difference disappears by adding the contributions of $\Xi^{0} \rightarrow \Lambda \pi^{0}$ and $\Xi^{-} \rightarrow \Lambda \pi^{-}$together. Therefore, we still have $D_{1 u}^{\Lambda, \Xi}(z)=D_{1 d}^{\Lambda, \Xi}(z)$. Other contributions are all isospin symmetric, so there is actually no isospin symmetry violation for $\Lambda$ production.

Polarized FFs. For polarized FFs, the formalism is very much similar to the unpolarized case. We only need to consider the spin transfer in the corresponding decay process, i.e.,

$$
\Delta D_{1 q}^{h, \mathrm{dec}}(z)=\sum_{h_{j}} \operatorname{Br}\left(h, h_{j}\right) \int d z^{\prime} K_{h, h_{j}}\left(z, z^{\prime}\right) t_{D}^{h, h_{j}}(z) \Delta D_{1 q}^{h_{j}}\left(z^{\prime}\right),
$$

where $t_{D}^{h, h_{j}}(z)$ is the spin transfer factor in the decay process. For polarized $\Lambda$ production, the only source for the violation is the weak decay $\Xi \rightarrow \Lambda \pi$. The spin transfer in this decay process is determined by the decay parameter $\gamma$ with an average value of $(1+2 \gamma) / 3$. However, the difference of the spin transfer for $\Xi^{0} \rightarrow \Lambda \pi^{0}$ and $\Xi^{-} \rightarrow \Lambda \pi^{-}$is very small, i.e., $2 \delta \gamma / 3 \sim 0.027$ [5]. Positivity bounds demand that the violation should be, in any case, less than this factor times $D_{1 u}^{\Lambda, \Xi}(z)$. Considering that $D_{1 u}^{\Lambda, \Xi}(z)$ is only a small fraction of $D_{1 u}^{\Lambda}(z)$, we conclude that the isospin symmetry violation in the polarized FFs of $\Lambda$ can only be a tiny effect.

\section{Fit to Belle $\Lambda$ polarization data}

Now we just assume the isospin symmetry for the polarized $\Lambda$ FF and study whether we can describe the Belle data. The Belle experiment has measured the $\Lambda$ transverse polarization w.r.t. the normal direction of the production plane which in TMD factorization can be expressed by the convolution of the polarized FF $D_{1 T q}^{\perp \Lambda}\left(z, p_{T}\right)$ with the unpolarized TMD FF. We take the Gaussian ansatz for TMD FFs, i.e., $D_{1 T q}^{\perp \Lambda}\left(z, p_{T}\right)=D_{1 T q}^{\perp \Lambda}(z) \frac{1}{\pi \Delta^{2}} \exp \left(-p_{T}^{2} / \Delta^{2}\right)$ with $D_{1 T q}^{\perp \Lambda}(z)=$ $N_{T q} \frac{\left(\alpha_{T q}+\beta_{T q}-1\right)^{\alpha_{T q}+\beta_{T q}-1}}{\left(\alpha_{T q}-1\right)^{\alpha_{T q}-1} \beta_{T q}^{\beta_{T q}}} z^{\alpha_{T q}}(1-z)^{\beta_{T q}} D_{1 q}^{\Lambda}(z)$. After integrating over the transverse momentum of the Lambda, the polarization is given by [4]

$$
P_{\Lambda}\left(z_{\Lambda}, z_{h}\right)=\frac{\sqrt{\pi} z_{h} \Delta}{2 z_{\Lambda} M_{\Lambda} \sqrt{z_{h}^{2}+z_{\Lambda}^{2} \Delta_{h}^{2} / \Delta^{2}}} \sum_{q}\left[R_{1 q}^{\Lambda h}\left(z_{\Lambda}, z_{h}\right) \frac{D_{1 T}^{\perp \Lambda}\left(z_{\Lambda}\right)}{D_{1 q}^{\Lambda}\left(z_{\Lambda}\right)}+(q \leftrightarrow \bar{q})\right] .
$$


The $R_{1 q}^{\Lambda h}$ factor corresponds to the relative weight of different flavors. We plot $R_{1 q}^{\Lambda \pi}$ using unpolarized FFs in Fig. 1(a), and it shows a clear difference between $\Lambda \pi^{+}$and $\Lambda \pi^{-}$especially in the small $z_{\Lambda}$ region. That might be a source for the different behaviors of the polarization. We also notice that the $\mathrm{c}$ quark contribution is not negligible. Therefore, we adopt the parameterization scheme to keep isospin symmetry, i.e., $u=d$, but leave other parameters free for fitting.

We show the fitting results in Fig 1(b). As we can see, the Belle Lambda polarization data can be described using polarized Lambda FFs with isospin symmetry. There is no need to introduce large isospin violation here. The fitted parameters are given in Tab. 1.

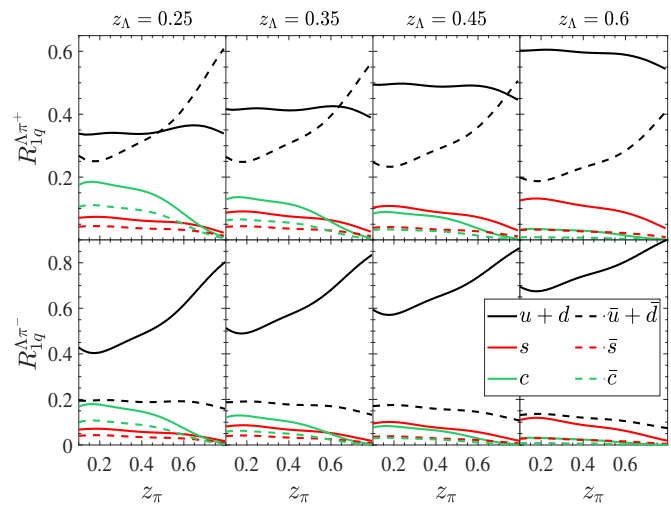

(a)

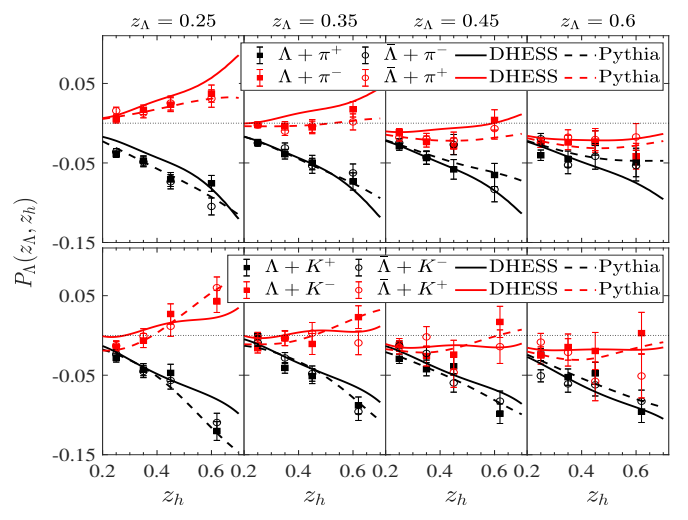

(b)

Figure 1: (a) The relative weight of different flavors in $e^{+} e^{-} \rightarrow \Lambda \pi X$. (b) Transverse polarization of $\Lambda$ in $e^{+} e^{-} \rightarrow \Lambda h^{ \pm} X$ compared with the Belle data.

Table 1: Parameters fitted to the Belle data. The first and second rows correspond to inputs of unpolarized FFs from DSV+DHESS and PythiA respectively. The values of $\Delta^{2} / \Delta_{h}^{2}$ are 0.951 and 0.939 respectively.

\begin{tabular}{c|cccccc}
\hline \hline parameter & $u, d$ & $s$ & $c$ & $\bar{u}, \bar{d}$ & $\bar{s}$ & $\bar{c}$ \\
\hline \multirow{2}{*}{$\frac{\Delta}{M_{\Lambda}} N_{T q}$} & 0.391 & -0.391 & 0.0278 & -0.456 & -0.430 & 0.401 \\
& 0.245 & -0.148 & 0.108 & -0.231 & 0.523 & -0.324 \\
\hline \multirow{2}{*}{$\alpha_{T q}$} & 1.38 & 6.91 & 1.43 & 1.00 & 2.64 & 11.6 \\
& 2.41 & 1.54 & 5.14 & 1.86 & 1.74 & 1.02 \\
\hline \multirow{2}{*}{$\beta_{T q}$} & 3.98 & 0.646 & 14.3 & 0.0319 & 2.77 & 14.9 \\
& 7.69 & 0.551 & 15.0 & 2.35 & 14.9 & 2.41 \\
\hline \hline
\end{tabular}

We further note that the isospin symmetry of $\Lambda$ FFs can be checked at the future SIDIS experiments. In Fig. 2(a)-(c), we show the predictions of the transverse polarizations of $\Lambda$ in $e p$, $e D$ and $e P b$ SIDIS [6]. It is clear that the isospin symmetric $\Lambda$ FFs [4] give almost identical polarizations for different nucleus targets, while the isospin violated FFs [2, 3] will generate significant shifts for the polarizations among different nucleus targets.

\section{Summary}

We make a systematic analysis of the isospin symmetry for FFs assuming isospin symmetry in strong interactions and considering hadron decay contributions. We show that the isospin symmetry 


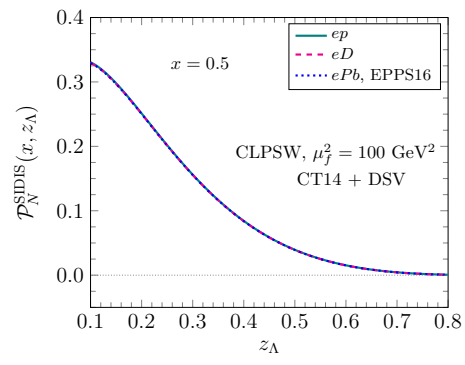

(a)

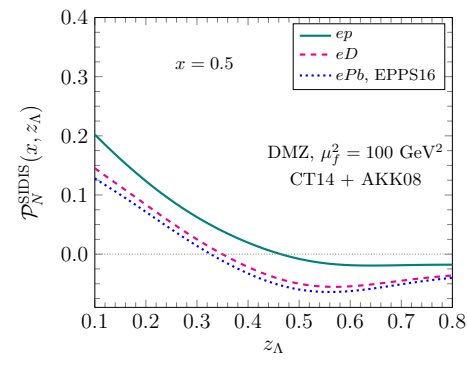

(b)

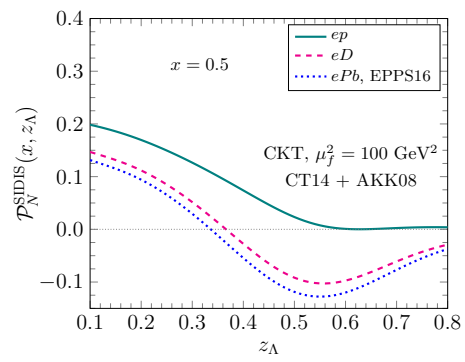

(c)

Figure 2: Predictions of the transverse polarizations of $\Lambda$ in $e p, e D$ and $e P b$ SIDIS using (a) isospin symmetric polarized $\Lambda$ FFs, (b) and (c) isospin violated FFs.

is held for unpolarized $\Lambda \mathrm{FFs}$, and only tiny violation for the polarized FFs. We demonstrate that the recent Belle data on the transverse polarization of $\Lambda$ can be reproduced under the isospin symmetric polarized $\Lambda$ FFs. The isospin symmetry of FFs can be checked in the future experiments on SIDIS with different nucleus targets.

\section{Acknowledgements}

The author is supported by the National Natural Science Foundation of China (Nos. 12005122, 11947055) and Shandong Province Natural Science Foundation Grant Nos. ZR2020QA082.

\section{References}

[1] Y. Guan et al. [Belle], Phys. Rev. Lett. 122, no.4, 042001 (2019) doi:10.1103/PhysRevLett.122.042001 [arXiv:1808.05000 [hep-ex]].

[2] U. D’Alesio, F. Murgia and M. Zaccheddu, Phys. Rev. D 102, no.5, 054001 (2020) doi:10.1103/PhysRevD.102.054001 [arXiv:2003.01128 [hep-ph]].

[3] D. Callos, Z. B. Kang and J. Terry, Phys. Rev. D 102, no.9, 096007 (2020) doi:10.1103/PhysRevD.102.096007 [arXiv:2003.04828 [hep-ph]].

[4] K. B. Chen, Z. T. Liang, Y. L. Pan, Y. K. Song and S. Y. Wei, Phys. Lett. B 816, 136217 (2021) doi:10.1016/j.physletb.2021.136217 [arXiv:2102.00658 [hep-ph]].

[5] P. A. Zyla et al. [Particle Data Group], PTEP 2020, no.8, $083 C 01$ (2020) doi:10.1093/ptep/ptaa104

[6] K. b. Chen, Z. t. Liang, Y. k. Song and S. y. Wei, [arXiv:2108.07740 [hep-ph]]. 